\title{
Prostate cancer of transition zone origin lacks TMPRSS2-ERG gene fusion
}

\author{
Charles C Guo, Geyan Zuo, Dongdong Cao, Patricia Troncoso and Bogdan A Czerniak* \\ Department of Pathology, The University of Texas M. D. Anderson Cancer Center, Houston, TX, USA
}

\begin{abstract}
Recent studies have shown a unique chromosomal rearrangement that leads to the fusion of $5^{\prime}$-transmembrane protein serine proteinase-2 (TMPRSS2) with the EST-related gene (ERG) in prostate cancer. In this study, we used fluorescence in situ hybridization to evaluate TMPRSS2-ERG gene fusion in prostate cancer of different zonal origins. Radical prostatectomy specimens with multifocal prostate cancer were obtained from 30 patients who were treated at our institution. Two separate tumor foci in each specimen, one in the peripheral zone and the other in the transition zone, were selected for gene fusion analysis. The selected peripheral zone tumor foci had a mean Gleason score of 6.8 (range, 6-7) and a mean tumor volume of $1.2 \mathrm{~cm}^{3}$ (range, $0.1-4.6 \mathrm{~cm}^{3}$ ). The selected transition zone tumor foci had a mean Gleason score of 6.7 (range, 5-8) and a mean tumor volume of $4.0 \mathrm{~cm}^{3}$ (range, $0.5-9.0 \mathrm{~cm}^{3}$ ). ERG gene rearrangement was not observed in any transition zone tumors; however, it was found in the peripheral zone tumors in 13 cases $(43 \%)$. In 10 cases, the rearrangement was associated with the deletion of the $5^{\prime}$-end of ERG. In conclusion, we found that TMPRSS2-ERG gene fusion is associated with the zonal origin of prostate cancer. This gene fusion is prevalent in prostate cancer arising from the peripheral zone, but is lacking in prostate cancer arising from the transition zone.
\end{abstract}

Modern Pathology (2009) 22, 866-871; doi:10.1038/modpathol.2009.57; published online 24 April 2009

Keywords: prostate cancer; transition zone; peripheral zone; TMPRSS2-ERG gene fusion; multifocal

Recent studies have shown that most prostate cancers have a unique chromosomal rearrangement in chromosome $21 .{ }^{1}$ This rearrangement is characterized by the fusion of $5^{\prime}$-transmembrane protein serine proteinase-2 (TMPRSS2) with an oncogene, EST-related gene $(E R G)$. Owing to the presence of androgen receptor-responsive elements in $5^{\prime}$ TMPRSS2, the TMPRSS2-ERG gene fusion leads to an aberrant function of oncogenic $E R G$, which is believed to play an important role in prostate cancer oncogenesis. TMPRSS2 can also fuse with other oncogenes, such as ETV1, ETV4, and ETV5, leading to prostate cancer, ${ }^{1-3}$ but TMPRSS2-ERG gene fusion is the most prevalent rearrangement.

Several studies have suggested that TMPRSS2$E R G$ gene fusion is associated with an aggressive phenotype of prostate cancer. Rajput et $a l^{4}$ reported that TMPRSS2-ERG gene fusion was more prevalent in moderately differentiated than in well-differentiated prostate cancer. Mehra et $a l^{5}$ reported that

${ }^{*}$ Correspondence: Dr B Czerniak, MD, PhD, Department of Pathology, Unit 085, The University of Texas M. D. Anderson Cancer Center, 1515 Holcombe Blvd., Houston, TX 77030-4009, USA.

E-mail: bczernia@mdanderson.org

Received 20 November 2008; revised 24 March 2009; accepted 25 March 2009; published online 24 April 2009
TMPRSS2-ERG gene fusion was statistically associated significantly with high pathological stage. Nam et $a l^{6}$ found that TMPRSS2-ERG expression was the single most significant predictor of disease relapse after surgery, independent of prostate-specific antigen (PSA) level, tumor grade, and tumor stage. However, Gopalan et $a l^{7}$ recently reported that TMPRSS2-ERG gene fusion was not associated with tumor pathological stage, biochemical recurrence, metastasis, or with the overall survival of the patient in a study of more than 500 patients. Therefore, the clinical significance of TMPRSS2-ERG gene fusion in prostate cancer remains uncertain.

The prostate is composed of three anatomic zones, namely peripheral zone, transition zone, and central zone. ${ }^{8-9}$ Although the majority of prostate cancers arise in the peripheral zone, many prostate cancers also develop in the transition zone. Compared with peripheral zone cancers, transition zone cancers tend to have a large tumor volume, a higher PSA level, and a lower Gleason score, and generally have a more favorable prognosis. ${ }^{10-13}$ Some studies have suggested that there are differential expression levels of proliferative genes and oncogenes between transition and peripheral zone tumors, which may contribute to their clinical and biological differences. ${ }^{14-17}$ However, to our knowledge, the role of TMPRSS2-ERG gene fusion in the zonal origin of 
prostate cancer has not been studied. In this study, we evaluated the TMPRSS2-ERG gene fusion in prostate cancers arising from the transition zone in comparison with those arising from the peripheral zone.

\section{Materials and methods}

\section{Case Selection and Pathological Evaluation}

We retrospectively searched our pathology file at The University of Texas M. D. Anderson Cancer Center (Houston, TX, USA) from 2001 to 2008, and selected 30 patients with multifocal prostate cancer in radical prostatectomy specimens on the basis of two criteria, namely (1) there were at least two independent tumor foci, as described earlier ${ }^{18}$ and (2) one tumor focus was located in the peripheral zone, and the other one was in the transition zone. When several tumor foci were present, the largest tumor focus from each zone was selected for the analysis. No patient had undergone radiation, hormone therapy, or transurethral prostatic resection before radical prostatectomy. Each tumor focus was graded according to the Gleason grading system. The volume of each tumor focus was calculated using a formula described earlier. ${ }^{18}$ The demographic and clinicopathologic features of the patients were obtained from medical records and from pathological examination of specimens.

\section{Determination of TMPRSS-ERG Gene Fusion}

TMPRSS2-ERG gene fusion was evaluated using break-apart fluorescence in situ hybridization (FISH). The break-apart probes, consisting of a rhodamine-labeled 5'-ERG probe (BAC RP11-95I21) and a fluorescein isothiocyanate-labeled $3^{\prime}$-ERG probe (BAC RP11-476D17), were obtained from the Children's Hospital of Oakland Research Institute (Oakland, CA, USA). Tissue pretreatment was performed using the Paraffin Pretreatment Kit I (Vysis, Des Plaines, IL, USA), and hybridization and washing were performed using Vysis hybridization reagents, following the manufacturer's protocols. We used the break-apart approach, resulting in two pairs of co-localized green and red signals in cells, with no rearrangement of ERG. In cells with TMPRSS2-ERG gene fusion, only one pair of colocalized green and red signals was maintained; the other broke into one green signal and one red signal. In cells with TMPRSS2-ERG gene fusion associated with the deletion of intervening DNA, one pair of colocalized green and red signals and one green signal were present-the $5^{\prime}$-region (red) signal was lost. The specificity and quality of the probes were confirmed by hybridization to the metaphase spread of the normal peripheral lymphocytes. A mean of 100 cells was evaluated per tumor focus.
Table 1 Summary of pathological features and TMPRSS2-ERG gene fusion status in peripheral zone and transition zone tumor foci

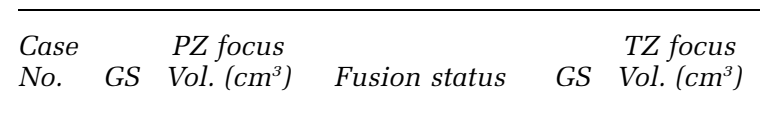

$\begin{array}{r}\text { status } \\ \hline\end{array}$

\begin{tabular}{|c|c|c|c|c|c|c|}
\hline 1 & $3+4$ & 0.8 & Pos. with no del. & $3+3$ & 0.8 & Neg. \\
\hline 2 & $3+4$ & 1.9 & Pos. with del. & $2+3$ & 3.8 & Neg. \\
\hline 3 & $3+4$ & 3.0 & Pos. with del. & $3+4$ & 2.8 & Neg. \\
\hline 4 & $3+4$ & $4.6^{\mathrm{a}}$ & Pos. with del. & $3+4$ & 7.2 & Neg. \\
\hline 5 & $3+4$ & 0.8 & Pos. with del. & $3+2$ & 2.4 & Neg. \\
\hline 6 & $3+4$ & 0.5 & Pos. with del. & $3+2$ & $1.7^{\mathrm{b}}$ & Neg. \\
\hline 7 & $3+4$ & 2.2 & Neg. & $3+4$ & 1.7 & Neg. \\
\hline 8 & $3+3$ & 1.4 & Pos. with del. & $3+3$ & 2.4 & Neg. \\
\hline 9 & $4+3$ & 1.8 & Pos. with no del. & $3+4$ & $4.9^{\mathrm{b}}$ & Neg. \\
\hline 10 & $3+4$ & 0.4 & Neg. & $3+4$ & $12.8^{\mathrm{a}, \mathrm{b}}$ & Neg. \\
\hline 11 & $3+3$ & 0.4 & Neg. & $3+3$ & 6.0 & Neg. \\
\hline 12 & $3+4$ & 1.6 & Neg. & $3+3$ & 2.4 & Neg. \\
\hline 13 & $3+3$ & 0.7 & Neg. & $3+4$ & 4.8 & Neg. \\
\hline 14 & $3+4$ & 1.1 & Pos. with no del. & $3+3$ & 0.6 & Neg. \\
\hline 15 & $3+4$ & 2.7 & Neg. & $3+4$ & 6.7 & Neg. \\
\hline 16 & $3+4$ & 0.6 & Neg. & $3+4$ & 0.5 & Neg. \\
\hline 17 & $3+4$ & $2.8^{\mathrm{a}}$ & Neg. & $3+4$ & 2.5 & Neg. \\
\hline 18 & $3+4$ & 0.5 & Neg. & $3+4$ & 3.6 & Neg. \\
\hline 19 & $3+3$ & 0.8 & Neg. & $3+4$ & 5.0 & Neg. \\
\hline 20 & $3+4$ & 0.3 & Pos. with del. & $4+3$ & 2.9 & Neg. \\
\hline 21 & $3+3$ & 1.6 & Neg. & $4+3$ & $9.0^{\mathrm{b}}$ & Neg. \\
\hline 22 & $3+4$ & 1.2 & Pos. with del. & $4+3$ & 6.0 & Neg. \\
\hline 23 & $4+3$ & 1.2 & Neg. & $3+4$ & 4.8 & Neg. \\
\hline 24 & $3+4$ & 0.2 & Pos. with del. & $3+3$ & 4.8 & Neg. \\
\hline 25 & $3+4$ & 0.4 & Neg. & $4+3$ & $6.0^{\mathrm{a}}$ & Neg. \\
\hline 26 & $3+3$ & 0.1 & Pos. with del. & $3+5$ & $4.7^{\mathrm{b}}$ & Neg. \\
\hline 27 & $3+4$ & 0.1 & Neg. & $4+4$ & $1.4^{\mathrm{a}, \mathrm{b}}$ & Neg. \\
\hline 28 & $4+3$ & 0.8 & Neg. & $4+3$ & $3.8^{\mathrm{a}, \mathrm{b}}$ & Neg. \\
\hline 29 & $4+3$ & 1.4 & Neg. & $3+5$ & 3.1 & Neg. \\
\hline 30 & $3+3$ & 0.1 & Neg. & $4+3$ & $1.8^{\mathrm{a}, \mathrm{b}}$ & Neg. \\
\hline
\end{tabular}

del., deletion; GS, Gleason score; neg., negative; pos., positive; PZ, peripheral zone; TZ, transition zone; vol., volume.

${ }^{\text {a }}$ Extraprostatic extension.

$\mathrm{b}^{\mathrm{b}}$ Tumor involving margin of resection.

\section{Results}

The mean age of patients in this study was 59 years (range, 46-71 years). Twenty-four were white, and six were black. The overall Gleason scores in radical prostatectomy specimens had a mean of 7.0 (range, $6-8$ ). There were a mean of 3.3 (range, 2-5) tumor foci in the specimens. Although the tumor was confined to the prostate in 23 cases, it extended into the extraprostatic adipose tissue in 7 cases (Table 1). In all cases, the tumor did not invade the seminal vesicles. In eight cases, the tumors involved the margin of resection. In addition, tumor metastasis to the lymph node was present in 1 of the 22 patients who underwent pelvic lymph node dissection.

Two separate tumor foci were selected for analysis from each radical prostatectomy specimen (Figure 1a). Prostatic adenocarcinoma in the transition zone typically showed large irregular glands lined by tall columnar cells with pale-to-clear cytoplasm and basally located nuclei (Figure 1b), whereas prostatic adenocarcinoma in the peripheral zone was characterized by small round glands lined by cuboidal 


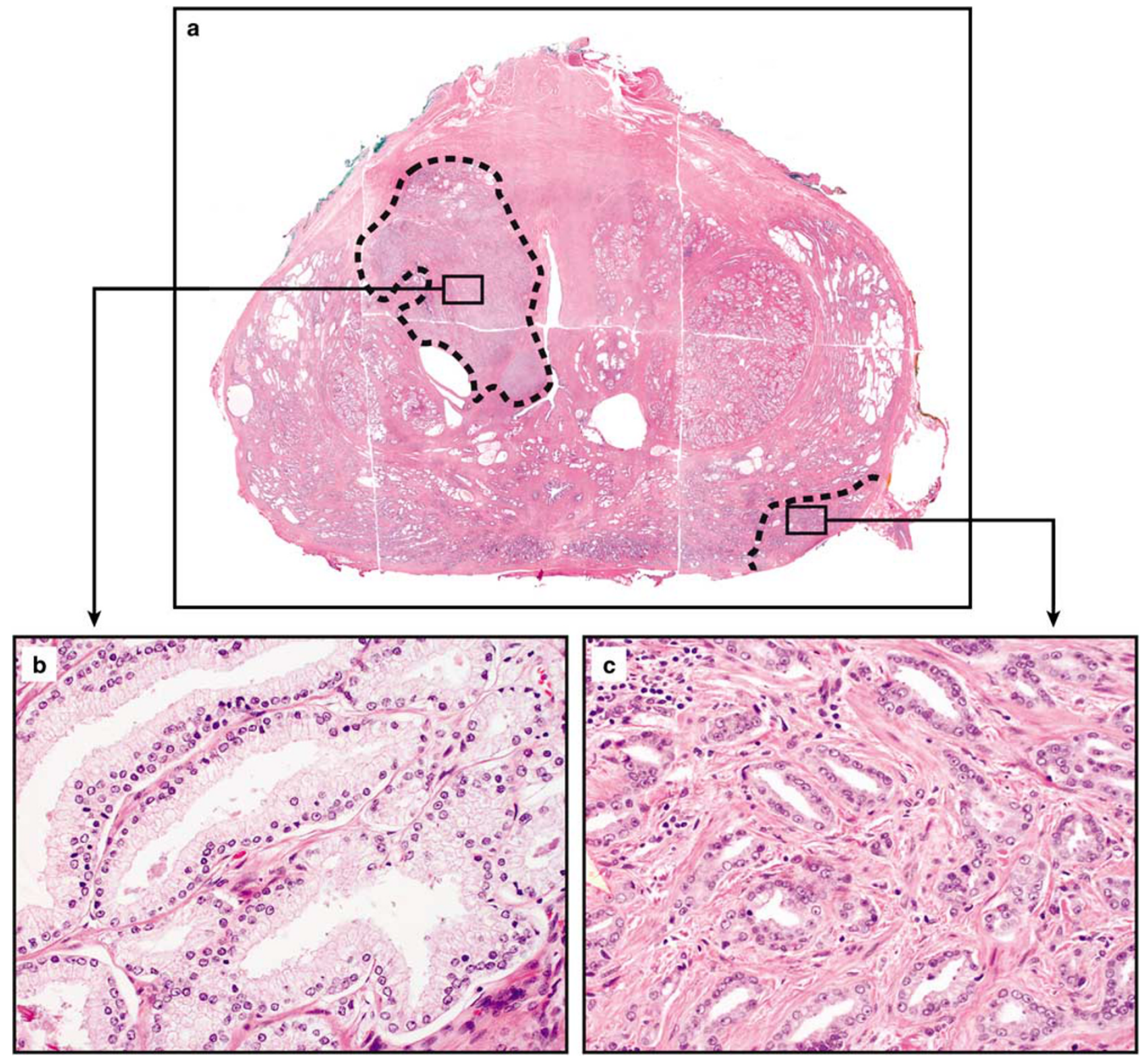

Figure 1 Prostatic adenocarcinoma of different zonal origins. (a) A transverse section through the mid-prostate shows two separate foci of prostatic adenocarcinoma, one in the left transition zone and the other in the right posterolateral peripheral zone. (b) Prostatic adenocarcinoma in the transition zone shows large irregular glands lined by tall columnar cells with pale-to-clear cytoplasm and basally located nuclei. (c) Prostatic adenocarcinoma in the peripheral zone shows small round glands lined by cuboidal cells with amphophilic cytoplasm and centrally located nuclei.

cells with amphophilic cytoplasm and centrally located nuclei (Figure 1c). The selected peripheral zone tumor foci had a mean Gleason score of 6.8 (range, 6-7) and a mean tumor volume of $1.2 \mathrm{~cm}^{3}$ (range, $0.1-4.6 \mathrm{~cm}^{3}$ ). The selected transition zone tumor foci had a mean Gleason score of 6.7 (range, $5-8$ ) and a mean tumor volume of $4.0 \mathrm{~cm}^{3}$ (range, $0.5-9.0 \mathrm{~cm}^{3}$ ). In 24 cases, the selected transition zone tumor focus was larger than the peripheral zone tumor focus.

TMPRSS2-ERG gene fusion was evaluated by FISH in both peripheral zone and transition zone tumor foci. In all cases, transition zone tumors showed normal signal patterns for $E R G$, with no gene rearrangement; the pattern was characterized by two pairs of co-localized green and red signals (Figure 2a). In 13 cases (43\%), peripheral zone tumors showed $E R G$ gene rearrangement, which was characterized by the break apart of one of the two colocalized signals. In 10 cases, ERG gene rearrangement was associated with the deletion of the $5^{\prime}$-end of ERG (red signal) (Figure 2b); the rearrangement was not associated with the deletion in the remaining three cases (Figure 2c).

A total of 24 patients were followed-up for a mean of 34.3 months (range, 8-82 months) after radical 

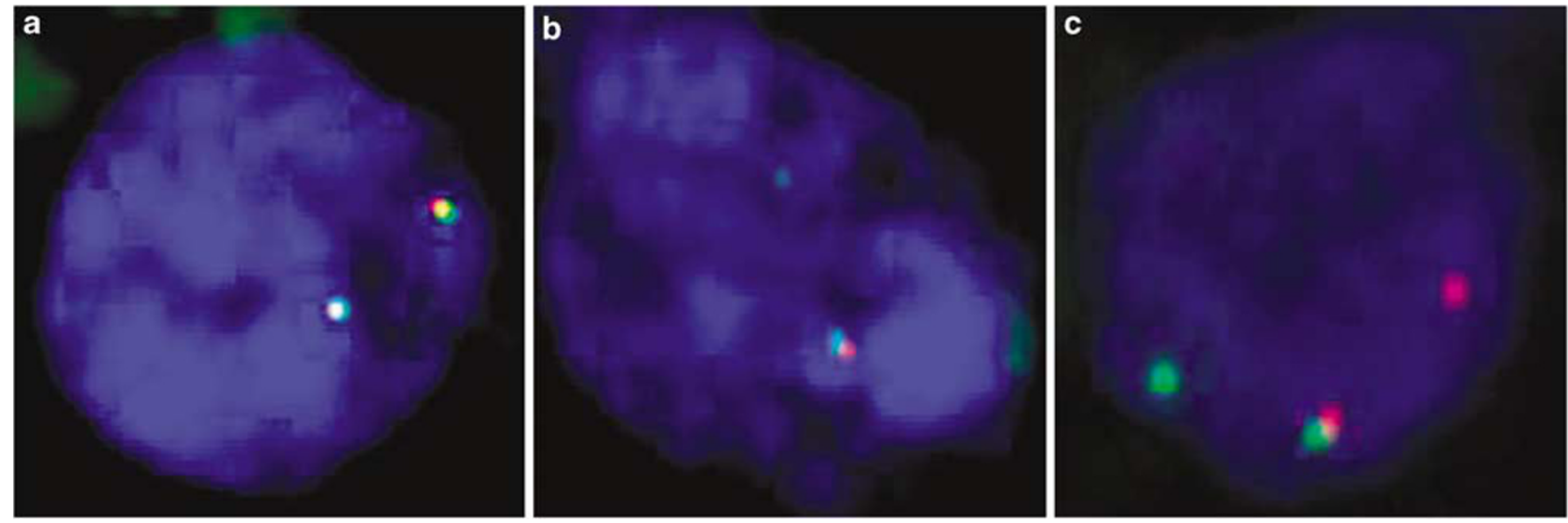

Figure $2 E R G$ gene rearrangement in prostatic adenocarcinoma of different zones. (a) Prostatic adenocarcinoma in the transition shows no $E R G$ gene rearrangement. (b) Prostatic adenocarcinoma in the peripheral zone shows $E R G$ gene rearrangement with the deletion of the $3^{\prime}$-region (red signal). (c) Prostatic adenocarcinoma in the peripheral zone shows $E R G$ gene rearrangement with no deletion.

prostatectomy; the remaining 6 patients were followed-up for $<6$ months. Twenty-two of the 24 patients were alive without evidence of disease. Of the remaining two patients, one experienced recurrent increase in PSA levels 21 months after surgery; the other patient presented with tumor metastasis to a lymph node at the time of surgery and subsequently received hormonal therapy with undetectable PSA 26 months after surgery. ERG gene rearrangement was not found in the studied tumor foci of either of the two patients.

\section{Discussion}

The zonal anatomy of the prostate was first described by McNeal. ${ }^{8,9}$ Using a three-dimensional model, McNeal divided the prostate into three anatomic zones, namely the peripheral zone, transition zone, and central zone. The peripheral zone forms the bulk of the posterior, lateral, and apical regions of the prostate, and accounts for $75 \%$ of the gland. The transition zone surrounds the proximal prostatic urethra and accounts for $5 \%$ of the gland in the absence of benign prostatic hyperplasia. The central zone forms a cone-shaped structure that constitutes the majority of the base of the prostate and accounts for $20 \%$ of the gland. The prostate is covered anteriorly by the anterior fibromuscular stroma. This zonal anatomy model of the prostate has been widely accepted.

Most $(75 \%)$ prostate cancers develop in the peripheral zone, but some $(20 \%)$ arise from the transition zone. ${ }^{10-13}$ The zonal origin of prostate cancer is largely determined on the basis of McNeal's model. The transition zone boundary, a thin layer of compressed fibromuscular tissue extending in an arc from the dorsal urethra to the anterior fibromuscular stroma, can sometimes be appreciated on examination with a low-magnification lens. ${ }^{9}$ In addition, histological features may help to establish the zonal origin of prostate cancer in small biopsy specimens. ${ }^{10}$ Transition zone prostate cancers often show large irregular glands with tall columnar cells, pale-to-clear cytoplasm, and basally located nuclei. In contrast, peripheral zone prostate cancers are characterized by small round glands with cuboidal cells, amphophilic cytoplasm, and centrally located nuclei.

However, one should be cautious in using the histological features to determine the zonal origin of prostate cancer. Garcia et $a l^{19}$ recently studied the so-called 'transitional-zone look', which is characterized by prostatic glands of variable sizes with tall columnar cells showing basally oriented nuclei and clear to pale pink cytoplasm, in different zonal origins of prostate cancer. They found that only half of transition zone cancers showed an extensive ( $>50 \%$ ) 'transition-zone look', although the 'transition-zone look' was more frequent in transition zone cancers than in peripheral zone cancers. Furthermore, they also observed a non-focal (>25\%) 'transition-zone look' in up to $35 \%$ of peripheral zone cancers. Therefore, the histological features alone are insufficient to determine the zonal origin of prostate cancer, especially in limited samples, such as prostate needle biopsy. In this study, we used the McNeal's zonal anatomy model to determine the zonal origin of prostate cancer.

Most studies have found that transition zone prostate cancer has a more favorable clinical course than peripheral zone prostate cancer. ${ }^{10-13}$ Although transition zone tumors often present with a larger volume and a higher PSA level than peripheral zone tumors, they tend to have a lower Gleason score. Shannon et $a l^{11}$ found that transition zone tumors were more likely to be organ-confined than peripheral zone tumors. Greene et $a l^{12}$ reported that transition zone tumors not only have a lower rate of extraprostatic extension but also a significantly lower rate of biochemical recurrence than peripheral zone cancers of the same tumor volume and grade. 
Furthermore, Noguchi et $a l^{13}$ found that the 5-year biochemical cure rate for transition zone tumors was $72 \%$ compared with $49 \%$ for peripheral zone tumors.

Several factors may contribute to the indolent clinical course of transition zone tumors. Anatomically, transition zone tumors are confined posterolaterally by the peripheral zone and anteriorly by the anterior fibromuscular stroma. Therefore, transition zone tumors are less likely to extend out of the prostate than peripheral zone tumors. Gene expression profiling using cDNA microarray techniques have shown that hundreds of genes have different expression levels in transition zone and peripheral zone tumors. ${ }^{14,15}$ Compared with peripheral zone tumors, transition zone tumors have a low proliferation rate and low microvessel density. ${ }^{16}$ In addition, transition zone tumors also express lower levels of p53 and bcl-2 than peripheral zone tumors. ${ }^{16,17}$ These intrinsic biological difference between transition zone and peripheral zone tumors may also contribute to their different clinical behaviors.

Most prostate cancers are multifocal. We earlier analyzed radical prostatectomy specimens through whole-mount processing and found that $83 \%$ (149 of 180) of prostate cancers had at least 2 tumor foci. ${ }^{18}$ Furthermore, we found that the tumor foci of both peripheral zone and transition zone origins were present in $52 \%$ of the cases. Recently, Arora et $a l^{20}$ found that $87 \%$ of prostate cancers were multifocal in the radical prostatectomy specimens, and only $9 \%$ of multifocal prostate cancers had all the tumor foci with the same primary and secondary Gleason grades. Cheng et $a 2^{21}$ analyzed microsatellite DNA alterations from separate tumor foci in the same prostate. Fifteen of 18 cases had discordant patterns of allelic loss in separate tumor foci. The histological and biological heterogeneity of multifocal tumors suggests that they arise independently within the same prostate gland.

Interestingly, TMPRSS2-ERG gene fusion also shows significant heterogenicity in multifocal prostate cancers. Barry et $a l^{22}$ reported that 13 of the 32 $(41 \%)$ radical prostatectomy specimens with multifocal prostate cancer showed discordant TMPRSS2$E R G$ gene fusion. Furthermore, in the specimens with a positive TMPRSS2-ERG fusion, $76 \%$ of the cases showed discordance in at least one focus. Mehra et $a l^{23}$ also reported that 20 of the $43(49 \%)$ multifocal prostate cancers had the discordant TMPRSS2-ERG gene fusion status. In our studies, 13 of the $30(43 \%)$ specimens showed the discordant TMPRSS-ERG gene fusion status. The heterogenicity of TMPRSS2-ERG gene fusion suggests that multifocal prostate cancer may arise from multiple independent clonal expansions.

Our study has some potential limitations. Owing to the small number of specimens, we could not show an association of TMPRSS2-ERG gene fusion with pathological stage, tumor volume, or Gleason score in peripheral zone tumors. Furthermore,
TMPRSS2-ERG gene fusion was not investigated in all tumor foci in the radical prostatectomy specimen. However, the aim of our study was to investigate the association between TMPRSS2-ERG gene fusion and the zonal origin of prostate cancer. The association of TMPRSS2-ERG gene fusion with pathological stage and Gleason score has been evaluated in several large studies. ${ }^{4,5}$ Similarly, the heterogenicity of TMPRSS2-ERG gene fusion in multifocal prostate cancer has also been investigated..$^{22,23}$ Nonetheless, further validation in large cohort studies is still needed.

In summary, we showed that TMPRSS2-ERG gene fusion was associated with the zonal origin of prostate cancer. This fusion was highly prevalent in prostate cancer arising from the peripheral zone, but was generally lacking in prostate cancer arising from the transition zone. The lack of TMPRSS2-ERG gene fusion in transition zone prostate cancer, suggests that there are genetic and biologic differences in prostate cancer of different zonal origins.

\section{Acknowledgement}

We thank Cindy Soto, BS, HT (ASCP) and Ina Prokhorova, MD, HT, HTL (ASCP) for their expert technical assistance with the pathological specimens.

\section{Conflict of interest}

The authors declare no conflict of interest.

\section{References}

1 Tomlins SA, Rhodes DR, Perner S, et al. Recurrent fusion of TMPRSS2 and ETS transcription factor genes in prostate cancer. Science 2005;310:644-648.

2 Tomlins SA, Mehra R, Rhodes DR, et al. TMPRSS2:ETV4 gene fusions define a third molecular subtype of prostate cancer. Cancer Res 2006;66:33963400 .

3 Helgeson BE, Tomlins SA, Shah N, et al. Characterization of TMPRSS2:ETV5 and SLC45A3:ETV5 gene fusions in prostate cancer. Cancer Res 2008;68:73-80.

4 Rajput AB, Miller MA, De Luca A, et al. Frequency of the TMPRSS2:ERG gene fusion is increased in moderate to poorly differentiated prostate cancers. J Clin Pathol 2007;60:1238-1243.

5 Mehra R, Tomlins SA, Shen R, et al. Comprehensive assessment of TMPRSS2 and ETS family gene aberrations in clinically localized prostate cancer. Mod Pathol 2007;20:538-544.

6 Nam RK, Sugar L, Yang W, et al. Expression of the TMPRSS2:ERG fusion gene predicts cancer recurrence after surgery for localised prostate cancer. Br J Cancer 2007;97:1690-1695.

7 Gopalan A, Leversha MA, Satagopan JM, et al. TMPRSS2-ERG gene fusion is not associated with outcome in patients treated by prostatectomy. Cancer Res 2009;69:1400-1406. 
8 McNeal JE. The zonal anatomy of the prostate. Prostate 1981;2:35-49.

9 McNeal JE. Normal histology of the prostate. Am J Surg Pathol 1988;12:619-633.

10 McNeal JE, Redwine EA, Freiha FS, et al. Zonal distribution of prostatic adenocarcinoma. Correlation with histologic pattern and direction of spread. Am J Surg Pathol 1988;12:897-906.

11 Shannon BA, McNeal JE, Cohen RJ. Transition zone carcinoma of the prostate gland: a common indolent tumour type that occasionally manifests aggressive behavior. Pathology 2003;35:467-471.

12 Greene DR, Wheeler TM, Egawa S, et al. A comparison of the morphological features of cancer arising in the transition zone and in the peripheral zone of the prostate. J Urol 1991;146:1069-1076.

13 Noguchi M, Stamey TA, Neal JE, et al. An analysis of 148 consecutive transition zone cancers: clinical and histological characteristics. J Urol 2000;163: 1751-1755.

14 Noel EE, Ragavan N, Walsh MJ, et al. Differential gene expression in the peripheral zone compared to the transition zone of the human prostate gland. Prostate Cancer Prostatic Dis 2008;11:173-180.

15 van der Heul-Nieuwenhuijsen L, Hendriksen PJ, van der Kwast TH, et al. Gene expression profiling of the human prostate zones. BJU Int 2006;98:886-897.

16 Erbersdobler A, Fritz H, Schnöger S, et al. Tumour grade, proliferation, apoptosis, microvessel density, p53, and $b c l-2$ in prostate cancers: differences between tumours located in the transition zone and in the peripheral zone. Eur Urol 2002;41:40-46.

17 Sakai I, Harada K, Hara I, et al. A comparison of the biological features between prostate cancers arising in the transition and peripheral zones. BJU Int 2005;96:528-532.

18 Chen ME, Johnston DA, Tang $\mathrm{K}$, et al. Detailed mapping of prostate carcinoma foci: biopsy strategy implications. Cancer 2000;89:1800-1809.

19 Garcia JJ, Al-Ahmadie HA, Gopalan A, et al. Do prostatic transition zone tumors have a distinct morphology? Am J Surg Pathol 2008;32:1709-1714.

20 Arora R, Koch MO, Eble JN, et al. Heterogeneity of Gleason grade in multifocal adenocarcinoma of the prostate. Cancer 2004;100:2362-2366.

21 Cheng L, Song SY, Pretlow TG, et al. Evidence of independent origin of multiple tumors from patients with prostate cancer. J Natl Cancer Inst 1998;90:233-237.

22 Barry M, Perner S, Demichelis F, et al. TMPRSS2-ERG fusion heterogeneity in multifocal prostate cancer: clinical and biologic implications. Urology 2007;70:630-633.

23 Mehra R, Han B, Tomlins SA, et al. Heterogeneity of TMPRSS2 gene rearrangements in multifocal prostate adenocarcinoma: molecular evidence for an independent group of diseases. Cancer Res 2007;67: 7991-7995. 